\title{
Impact of an electric field on P-type ATPases
}

\author{
Christian Weidemüller and Karin Hauser* \\ Institut für Biophysik, Johann Wolfgang Goethe-Universität Frankfurt, 60438 Frankfurt, Germany
}

\begin{abstract}
P-type ATPases are membrane proteins acting as ion pumps that drive an active transport of cations across the membrane against a concentration gradient. The required energy for the ion transport is provided by binding and hydrolysis of ATP. A reaction mechanism of ion transport and energy transduction is assumed to be common for all P-type ATPases and generally described by the Post-Albers cycle. Transient currents and charge translocation of P-type ATPases were extensively investigated by electrical measurements that apply voltage jumps to initiate the reaction cycle. In this study, we simulate an applied voltage across the membrane by an electric field and perform electrostatic calculations in order to verify the experimentally-driven hypothesis that the energy transduction mechanism is regulated by specific structural elements. Side chain conformational and ionization changes induced by the electric field are evaluated for each transmembrane helix and the selectivity in response is qualitatively analyzed for the $\mathrm{Ca}^{2+}$-ATPase as well as for structural models of the $\mathrm{Na}^{+} / \mathrm{K}^{+}$-ATPase. Helix M5 responds with more conformer changes as compared to the other transmembrane helices what is even more emphasized when the stalk region is included. Thus our simulations support experimental results and indicate a crucial role for the highly conserved transmembrane helix M5 in the energy transduction mechanism of P-type ATPases.
\end{abstract}

Keywords: P-type ATPase, energy transduction, transport, electric field, electrostatics

\section{Introduction}

P-type ATPases are found in all branches of life and form a major class of ion pumps that are essential for many fundamental processes in biology [1]. The P-type ATPase family contains more than fifty members with various functions ranging from the generation of membrane potential to the removal of toxic ions from plant cells [2]. P-type ATPases are integral membrane proteins that share structural similarities as they contain a transmembrane region with $\alpha$-helices that is connected to the cytoplasmic head part. To fulfil their functions, the P-type ATPases use the energy of ATP that is bound and hydrolysed at the nucleotide binding domain located in the cytoplasmic part. In order to drive the active transport of cations through the transmembrane part, the energy provided at the cytoplasmic nucleotide binding site has to be transferred to the transmembrane domain where the ion transport takes place. This basic reaction mechanism is shared by all P-type ATPases and is commonly described with the classical E1/E2 model (Post-Albers cycle) [3,4] with two main conformational enzyme states, E1 and E2. Prominent members of the P-type ATPase family are the $\mathrm{Ca}^{2+}$-ATPase and the $\mathrm{Na}^{+} / \mathrm{K}^{+}$-ATPase. The $\mathrm{Ca}^{2+}$-ATPase is responsible for the relaxation of the muscle and the $\mathrm{Na}^{+} / \mathrm{K}^{+}$-ATPase for the generation of the membrane potential.

In the case of the $\mathrm{Ca}^{2+}$-ATPase, two $\mathrm{Ca}^{2+}$ ions are pumped out of the sarcoplasmic reticulum (SR) where the calcium is stored after the contraction of the muscle and 2-3 protons are countertransported per cycle [5]. The $\mathrm{Ca}^{2+}$-ATPase consists of four domains. The cytoplasmic part is divided in the phosphorylation domain (P-domain) containing the highly conserved sequence that play a key role in the

\footnotetext{
* Corresponding author: K. Hauser, Institut für Biophysik, Johann Wolfgang Goethe-Universität Frankfurt, Max-von-LaueStr. 1, 60438 Frankfurt, Germany. Tel.: +49 69798 46407; Fax: +49 6979846 423; E-mail: hauser@ biophysik.uni-frankfurt.de.
} 
phosphorylation of the enzyme, the nucleotide binding domain (N-domain) where the ATP binding takes place and the actuator domain (A-domain) which is responsible for the regulation of the binding and the release of $\mathrm{Ca}^{2+}$ ions. The transmembrane domain consists of ten $\alpha$-helices (M1-M10) extended by the so called stalk-region (S2-S5) that connects the transmembrane domain with the cytoplasmic part [6, 7]. The $\mathrm{Ca}^{2+}$-ATPase is predestinated for computational analysis since atomic coordinates have been determined for different enzyme states with high resolution [8-10]. The available X-ray structures of the $\mathrm{Ca}^{2+}$-ATPase show that a large conformational change between the E1 and the E2 enzyme states is necessary for the reaction mechanism [11].

In the case of the $\mathrm{Na}^{+} / \mathrm{K}^{+}$-ATPase, three $\mathrm{Na}^{+}$ions are pumped out of the cell to the cytoplasm while two $\mathrm{K}^{+}$ions are pumped in the opposite direction [12]. The pump activity creates an electrical gradient across the cell membrane which is important to maintain the membrane potential of cells. The $\alpha$-subunit of the $\mathrm{Na}^{+} / \mathrm{K}^{+}$-ATPase is referred as the catalytic subunit mediating ATP hydrolysis and ion transport. Similar to the $\mathrm{Ca}^{2+}$-ATPase, it also contains ten helices M1-M10 forming the transmembrane domain $[13,14]$. The sequence identity between the $\mathrm{Na}^{+} / \mathrm{K}^{+}$-ATPase and the $\mathrm{Ca}^{2+}$-ATPase is about $40 \%$ in the transmembrane domain $[15,16]$. Because of the high homology, it is possible to get a structural picture of the $\alpha$-subunit of the $\mathrm{Na}^{+} / \mathrm{K}^{+}$-ATPase and to construct decent models based on the solved coordinates of the $\mathrm{Ca}^{2+}$-ATPase. Voltage clamp fluorometry experiments on the $\mathrm{Na}^{+} / \mathrm{K}^{+}$-ATPase induce the reaction cycle by voltage jumps. It was shown that the transition between the main enzyme conformations (E1P and E2P) is coupled to a conformational change of transmembrane helix M5 and concluded that this highly conserved helix M5 may act as energy transduction element [17].

In this study, we analyze the energy transduction in P-type ATPases and the role of the helix M5 for coupling ATP hydrolysis to ion transport by multiconformation continuum electrostatic calculations (MCCE) [18-20]. Calculations were carried out with atomic coordinates of several X-Ray structures of the $\mathrm{Ca}^{2+}$-ATPase and modeled structures for the $\mathrm{Na}^{+} / \mathrm{K}^{+}$-ATPase. According to voltage clamp experiments, we simulated an electric field over the transmembrane region by an "ionic capacitor". Selectivity in helix response to the electric field was studied by side chain conformational and ionization changes in order to analyze the role of the helix M5 in the energy transduction mechanism of P-type ATPases in general.

\section{Methods}

Multiconformation continuum electrostatics (MCCE) combines traditional continuum electrostatics with molecular mechanics. The surrounding solvent is treated as a continuum whereas the protein flexibility is implemented by adding various side chain orientations (rotamers) to the rigid structure. The backbone remains fixed. Several conformers are generated for each residue differing by the ionization state, side chain orientation (rotamer) and hydrogen position. Since side chain rotamers are explicitly considered, the protein dielectric constant is kept low $(\varepsilon=4)$. A dielectric constant of $\varepsilon=80$ is used for the solvent. All electrostatic interactions are calculated with the Poisson-Boltzmann solver program DelPhi [21]. PARSE parameters are used for the atomic charges and radii [22] and Lennard-Jones parameters have been previously reported $[23,24]$. Protein microstates are created by choosing one conformer per residue. Those microstates are subjected to Monte Carlo sampling. The occupancy of each conformer is calculated as a function of $\mathrm{pH}$ according to a Boltzmann distribution of states. A detailed description of the MCCE method can be found elsewhere [24,25]. Our calculations were performed with program version 1.0. 
The electric field was simulated by an "ionic capacitor". This capacitor was realized by adding ions in a row above and below the transmembrane region of the protein. Different electric fields ranging from $6 \times 10^{9} \mathrm{~V} / \mathrm{m}$ to $1.6 \times 10^{12} \mathrm{~V} / \mathrm{m}$ were applied by varying the number of added ions. The response of the transmembrane helices M1-M10 were analyzed by calculating the occupancy of each conformer and comparing the difference in occupancy with and without the applied electric field. In order to consider perturbations induced by the presence of the capacitor ions, all calculations were performed with the capacitor setup, i.e. with ions when the electric field was applied and with neutral atoms in the absence of the field. The impact of the electric field was analyzed by the number of changes in conformer occupancy on each of the transmembrane helices. The evaluation was done with and without involvement of the stalk region S2-S5. The conformer changes were distinguished in ionization and rotamer changes. The latter were further differentiated in small and large structural changes, whereas a large structural change was classified as rotating a rotatable bond by more than $5^{\circ}$.

The transmembrane region was assigned with the structural classification program SCOP [26]. For the $\mathrm{Ca}^{2+}$-ATPase, calculations were performed with the calcium-bound state E1 (pdb entry: 1SU4) [9]. All crystal water molecules and inhibitors were removed in the coordinate files. Structural models were generated for the $\mathrm{Na}^{+} / \mathrm{K}^{+}$-ATPase with the programs ModWeb and 3D-Jigsaw [27] based on $\mathrm{Ca}^{2+}$-ATPase coordinates as templates (pdb entries: 1SU4 and 1WPG). Three protein sequences of the $\alpha$-subunit of the $\mathrm{Na}^{+} / \mathrm{K}^{+}$-ATPase were used to generate the three different models, i.e. the sequences of ovis aries (Swiss-prot entry: P04074) [28], of rattus norvegicus (Swiss-prot entry: Q64541) [29] and of torpedo california (Swiss-prot entry: P05025) [30].

A POPC(1-Palmitoyl 2-Oleoyl Phosphatidylcholin)-membrane was generated with VMD, program version 1.8.6 [31] and each protein structure was embedded in the membrane. The gap between the protein and the membrane was reduced by molecular dynamics (MD) simulations with runtimes between $50 \mathrm{ps}$ and $1 \mathrm{~ns}$. MD simulations were performed with the program NAMD, program version 2.6 [32]. The membrane model was parameterized in MCCE without charges and no rotamers. The whole simulation setup of protein, membrane, and capacitor was subjected to MCCE calculations at physiological $\mathrm{pH}=7$.

\section{Results and discussion}

The impact of an electric field on the transmembrane helices M1-M10 was first analyzed for the $\mathrm{Ca}^{2+}$ ATPase. Each transmembrane helix consists of about 20 residues as listed in Table 1. The extensions of the helices M2-M5 from the transmembrane region to the cytoplasmic phosphorylation site are termed as stalk-region that consists of two shorter helix extensions, S2 and S3 with 12 resp. 6 residues, and two longer links S4 and S5 with 18 resp. 19 residues. Furthermore, the fraction of ionizable residues is quoted in Table 1 for each helix. To avoid local electrostatic and steric interactions between the capacitor and the protein and in order to verify the influence of the chosen capacitor geometry, different capacitor setups were tested. Especially the ionizable residues on the helix ends and close to the ionic plates might change their ionization state if the distance to the capacitor plate varies.

Besides the geometry of the ionic capacitor, the strength of the applied field had to be chosen in that way that conformer changes are induced at all. Since the backbone remains fixed in the MCCE approach, potential conformer changes are limited by the subjected side chain rotamers. Thus, higher field strengths are expected to be necessary as compared to physiological and experimentally applied fields $\left(\sim 4.0 \times 10^{7} \mathrm{~V} / \mathrm{m}\right)$. We had to increase the electric field to $2.4 \times 10^{11} \mathrm{~V} / \mathrm{m}$ in order to get a distinctive 
Table 1

Impact of the electric field on the $\mathrm{Ca}^{2+}$-ATPase

\begin{tabular}{llcccccccccc}
\hline & & M1 & M2 & M3 & M4 & M5 & M6 & M7 & M8 & M9 & M10 \\
\hline TM & no of res & 21 & 21 & 20 & 18 & 20 & 21 & 23 & 20 & 19 & 21 \\
& [no of ioniz res] & {$[5]$} & {$[3]$} & {$[4]$} & {$[2]$} & {$[3]$} & {$[1]$} & {$[1]$} & {$[1]$} & {$[0]$} & {$[4]$} \\
SR & no of res & - & 12 & 6 & 18 & 19 & - & - & - & - & - \\
& [no of ioniz res] & - & {$[4]$} & {$[0]$} & {$[3]$} & {$[3]$} & - & - & - & - & - \\
TM & conformer changes & 4 & 3 & 5 & 2 & 5 & 2 & 5 & 5 & 2 & 6 \\
& rotamer changes & 1 & 3 & 1 & 1 & 4 & 1 & 0 & 1 & 0 & 0 \\
\multirow{3}{*}{ TM \& SR } & conformer changes & 4 & 9 & 9 & 9 & 13 & 2 & 5 & 5 & 2 & 6 \\
& rotamer changes & 1 & 6 & 5 & 4 & 9 & 1 & 0 & 1 & 0 & 0 \\
\hline
\end{tabular}

The total number of residues and in brackets the fraction of ionizable residues are listed in the upper rows for the transmembrane (TM) and the stalk (SR) region of each helix (M1-M10). The lower rows show the results of representative calculations indicating the number of residues that change their conformer upon application of the electric field. Significant rotamer changes have been extracted separately from the total number of conformer changes.

response in the simulations reflected by conformer changes of the protein side chains. For the alignment of the membrane to the protein, the runtime of the MD-simulations was varied. An increased runtime reduces the solvent accessible surface of the helix residues that are located at the protein-membrane interface.

The ionization and rotamer changes of the transmembrane helices recorded in the simulations might be influenced by the extent of solvent accessibility or the capacitor geometry. When changing the solvent accessibility, ionizable residues of the helices M1, M3 and M10 are influenced in particular. It turned out that these helices have no or less significant rotamer changes. Helix M8 is influenced by both, the solvent accessibility and the capacitor geometry. It is striking that the number of conformer changes of helix M5 is quite insensitive to setup variations and is sustained as compared to the other transmembrane helices. The conformer changes on M5 consist of several significant rotamer changes. That might be an indication for a stronger polarizability of helix M5 by an electric field, although the backbone is kept fixed in our simulation method.

It is not unexpected that the stalk-region may play an important role for the energy transduction because it connects the cation binding site with the cytoplasmic nucleotide binding and phosphorylation sites [33]. Therefore, the conformer changes induced by the electric field were analyzed for S2-S5 as well. The number of conformer changes increases significantly for the helices M2-M5 that are extended by the stalk part. Differentiating the conformer changes in ionization and rotamer changes (Table 1), the impact of the electric field can be analyzed in more detail. The absolute number of significant rotamer changes of helices M3 and M4 is only about half as compared to helix M5 (5 resp. 4 versus 9). Furthermore, these changes are concentrated at the helix ends whereas on helix M5 they are distributed along the whole helix. Helix M2 shows 6 significant rotamer changes when the stalk region is included in the evaluation and 3 conformer changes are attributed to ionization changes. M2 possesses 7 and thus the most ionizable residues among the helices with stalk region, 3 are located in the transmembrane part, 4 in the stalk part. 6 out of 7 ionizable residues are ionized at $\mathrm{pH} 7$ whereas 3 are already ionized without the field and 3 are getting ionized by the field. This high number of ionized residues might contribute to induce rotamer changes, especially rotamer changes of polar and ionizable residues.

The simulation setup was transferred from the $\mathrm{Ca}^{2+}$-ATPase to the constructed structural models of the $\mathrm{Na}^{+} / \mathrm{K}^{+}$-ATPase. The same electric field and geometry of the capacitor were used to evaluate changes in conformer occupancies. Three different protein sequences were chosen for the construction of the 
Table 2

Impact of the electric field on different structural models of the $\mathrm{N}^{+} / \mathrm{K}^{+}$-ATPase

\begin{tabular}{lccccccccccc}
\hline & & M1 & M2 & M3 & M4 & M5 & M6 & M7 & M8 & M9 & M10 \\
\hline P04074 & TM & 5 & 4 & 2 & 0 & 8 & 7 & 6 & 4 & 4 & 5 \\
& TM \& SR & 5 & 6 & 3 & 2 & 17 & 7 & 6 & 4 & 4 & 5 \\
Q64541 & TM & 6 & 2 & 4 & 1 & 9 & 5 & 5 & 6 & 5 & 3 \\
& TM \& SR & 6 & 4 & 6 & 5 & 18 & 5 & 5 & 6 & 5 & 3 \\
P05025 & TM & 4 & 0 & 4 & 2 & 6 & 4 & 6 & 5 & 4 & 4 \\
& TM \& SR & 4 & 1 & 5 & 13 & 15 & 4 & 6 & 5 & 4 & 4 \\
\hline
\end{tabular}

The number of residues that change their conformer upon application of the electric field are listed for representative calculations of the different protein sequences (P04074, Q64541, P05025). It is differentiated between the transmembrane region solely (TM) and the transmembrane region extended by the stalk region (TM \& SM).

models. The P04074 sequence of ovis aries was selected in accordance to the motivating experiments [17], the Q64541 sequence of rattus norvegicus because other voltage clamp experiments were carried out with this species [34], and the P05025 sequence of torpedo californica because of its diversity to the previous sequences. This diversity was determined with a linear alignment in BLAST [35]. Different enzyme states (E1 and E1P) of the $\mathrm{Ca}^{2+}$-ATPase were chosen as template for the construction of the modeled structures.

Table 2 lists the number of conformer changes induced by the electric field for all three structural models. The number of conformer changes has been evaluated for each helix with and without including the stalk region. Calculations with varying capacitor geometry revealed that the number of conformer changes can be influenced by the properties of the simulation setup for the helices M1, M6, M7 and M8 whereas the number remains stable for M5. Although this evaluation gives only qualitative indications based on modeled structures, it also becomes obvious that the number of conformer changes induced by the electric field is significantly higher for helix M5 than for the other helices, in particular when the stalk region is considered. As for the $\mathrm{Ca}^{2+}$-ATPase, these conformer changes are interpreted as a selective activation by the electric field.

Taken together, among the transmembrane helices, M5 reveals the most conformer (ionization and rotamer) changes induced by an electric field in the E1 state of the $\mathrm{Ca}^{2+}$-ATPase and structural models of the $\mathrm{N}^{+} / \mathrm{K}^{+}$-ATPase. If the stalk region is included in the analysis, the number of conformer changes on M5 increases extremely emphasizing the selective activation of M5 even more. The importance of helix M5 for the reaction mechanism of the $\mathrm{Ca}^{2+}$-ATPase and the $\mathrm{N}^{+} / \mathrm{K}^{+}$-ATPase has also been shown by spectroscopic studies. NMR studies revealed a flexibility of regions within the M5 segment that is necessary for ion binding and occlusion during the active transport [36,37]. Conformational changes of the $\mathrm{Ca}^{2+}$-ATPase upon calcium binding and release were monitored by infrared and fluorescence studies. It was concluded that the calcium binding affects the secondary structure of only a few residues being sufficient to conduct large scale changes of entire protein domains [38]. $\alpha$-Helical structures seem to be involved in the conformational changes during the phosphoenzyme conversion and calcium release reaction [39]. The analogue transition $\mathrm{E}_{1} \mathrm{P}-\mathrm{E}_{2} \mathrm{P}$ was studied for the $\mathrm{Na}^{+} / \mathrm{K}^{+}$-ATPase with voltage clamp fluorometry experiments [17] revealing molecular rearrangements of the M5-M6 loop. This extracellular M5-M6 loop and the attached helix M5 are highly conserved among all P-type ATPases [15,16]. Helix M5 extends from the cytoplasmic site of ATP hydrolysis into the cation binding region and thus might likely be crucial for coupling ATP hydrolysis to ion transport. 


\section{Conclusion}

Within the limitations of our simulation techniques, we could qualitatively show for the $\mathrm{Ca}^{2+}$-ATPase and structural models of the $\mathrm{Na}^{+} / \mathrm{K}^{+}$-ATPase that helix M5 is selectively activated by an electric field. This helix is highly conserved and is likely to act as energy transducing element in the ion transporting reaction mechanism of P-type ATPases. Future studies, combining MD simulations with electrostatic calculations, will be carried out in order to overcome method limitations in conformational dynamics.

\section{Acknowledgement}

We thank Marilyn Gunner (City College of New York) for providing us with the MCCE code and Ernst Bamberg (Max Planck Institute of Biophysics, Frankfurt) for valuable and encouraging discussions about energy transduction in P-type ATPases. Cluster access at the Frankfurt Center of Scientific Computing (CSC) and financial support of the Deutsche Forschungsgemeinschaft (SFB 472) is gratefully acknowledged.

\section{References}

[1] W. Kühlbrandt, Biology, structure and mechanism of P-type ATPases, Nature 5 (2004), 282-295.

[2] M.G. Palmgren and K.B. Axelsen, Evolution of P-type ATPases, Biochimica et Biophysica Acta - Bioenergetics 1365 (1998), 37-45.

[3] R.L. Post, S. Kume and C. Hegyvary, Activation by adenosine-triphosphate in phosphorylation kinetics of sodium and potassium ion transport adenosine-triphosphatase, Journal of Biological Chemistry 247 (1972), 6530-6540.

[4] R.W. Albers, Biochemical aspects of active transport, Annual Review of Biochemistry 36 (1967), 727-756.

[5] J.V. Moller, B. Juul and M. leMaire, Structural organization, ion transport, and energy transduction of P-type ATPases, Biochimica et Biophysica Acta - Reviews on Biomembranes 1286 (1996), 1-51.

[6] C. Toyoshima and G. Inesi, Structural basis of ion pumping by $\mathrm{Ca}^{2+}$-ATPase of the sarcoplasmic reticulum, Annual Review of Biochemistry 73 (2004), 269-292.

[7] D.L. Stokes and N.M. Green, Structure and function of the calcium pump, Annual Review of Biophysics and Biomolecular Structure 32 (2003), 445-468.

[8] K. Obara, N. Miyashita, C. Xu, I. Toyoshima, Y. Sugita, G. Inesi and C. Toyoshima, Structural role of countertransport revealed in $\mathrm{Ca}^{2+}$ pump crystal structure in the absence of $\mathrm{Ca}^{2+}$, Proceedings of the National Academy of Sciences of the United States of America 102 (2005), 14489-14496.

[9] C. Toyoshima, M. Nakasako, H. Nomura and H. Ogawa, Crystal structure of the calcium pump of sarcoplasmic reticulum at 2.6 A resolution, Nature 405 (2000), 647-655.

[10] T.L.M. Sorensen, J.V. Moller and P. Nissen, Phosphoryl transfer and calcium ion occlusion in the calcium pump, Science 304 (2004), 1672-1675.

[11] C. Toyoshima and H. Nomura, Structural changes in the calcium pump accompanying the dissociation of calcium, Nature 418 (2002), 605-611.

[12] J.H. Kaplan, Biochemistry of Na,K-ATPase, Annual Review of Biochemistry 71 (2002), 511-535.

[13] J.B. Lingrel and T. Kuntzweiler, $\mathrm{Na}^{+}, \mathrm{K}^{+}$-ATPase, Journal of Biological Chemistry 269 (1994), 19659-19662.

[14] P.L. Jorgensen, K.O. Hakansson and S.J.D. Karlish, Structure and mechanism of Na,K-ATPase: Functional sites and their interactions, Annual Review of Physiology 65 (2003), 817-849.

[15] H. Ogawa and C. Toyoshima, Homology modeling of the cation binding sites of $\mathrm{Na}^{+} \mathrm{K}^{+}$-ATPase, Proceedings of the National Academy of Sciences of the United States of America 99 (2002), 15977-15982.

[16] K.J. Sweadner and C. Donnet, Structural similarities of Na,K-ATPase and SERCA, the $\mathrm{Ca}^{2+}$-ATPase of the sarcoplasmic reticulum, Biochemical Journal 356 (2001), 685-704.

[17] S. Geibel, J.H. Kaplan, E. Bamberg and T. Friedrich, Conformational dynamics of the $\mathrm{Na}^{+} / \mathrm{K}^{+}$-ATPase probed by voltage clamp fluorometry, Proceedings of the National Academy of Sciences of the United States of America 100 (2003), 964969. 
[18] K. Hauser and A. Barth, Side-chain protonation and mobility in the sacroplasmic reticulum $\mathrm{Ca}^{2+}$-ATPase: Implication for proton countertransport and $\mathrm{Ca}^{2+}$ release, Biophysical Journal 93 (2007), 3259-3270.

[19] K. Hauser, J. Mao and M.R. Gunner, pH dependence of heme electrochemistry in cytochromes investigated by multiconformation continuum electrostatic calculations, Biopolymers 74 (2004), 51-54.

[20] J.J. Mao, K. Hauser and M.R. Gunner, How cytochromes with different folds control heme redox potential, Biochemistry 42 (2003), 9829-9840.

[21] A. Nicholls and B. Honig, A rapid finite-difference algorithm, utilizing successive over-relaxation to solve the PoissonBoltzmann equation, Journal of Computational Chemistry 12 (1991), 435-445.

[22] D. Sitkoff, K.A. Sharp and B. Honig, Accurate calculation of hydration free-energies using macroscopic solvent models, Journal of Physical Chemistry 98 (1994), 1978-1988.

[23] R.E. Georgescu, E.G. Alexov and M.R. Gunner, Combining conformational flexibility and continuum electrostatics for calculating $\mathrm{pK}_{a}$ s in proteins, Biophysics Journal 83 (2002), 1731-1748.

[24] E.G. Alexov and M.R. Gunner, Incorporating protein conformational flexibility into the calculation of pH-dependent protein properties, Biophysical Journal 72 (1997), 2075-2093.

[25] M.R. Gunner and E. Alexov, A pragmatic approach to structure based calculation of coupled proton and electron transfer in proteins, Biochimica et Biophysica Acta 1458 (2000), 63-87.

[26] A.G. Murzin, S.E. Brenner, T. Hubbard and C. Chothia, SCOP: A structural classification of proteins database for the investigation of sequences and structures, Journal of Molecular Biology 247 (1995), 536-540.

[27] P.A. Bates, L.A. Kelley, R.M. MacCallum and M.J.E. Sternberg, Enhancement of protein modeling by human intervention in applying the automatic programs 3D-JIGSAW and 3D-PSSM, Proteins - Structure Function and Genetics 45 (2001), 39-46.

[28] G.E. Shull, A. Schwartz and J.B. Lingrel, Amino-acid-sequence of the catalytic subunit of the $\left(\mathrm{Na}^{+}+\mathrm{K}^{+}\right)$-ATPase deduced from a complementary DNA, Nature 316 (1985), 691-695.

[29] O.I. Shamraj and J.B. Lingrel, A putative 4th $\mathrm{Na}^{+}, \mathrm{K}^{+}$-ATPase alpha-subunit gene is expressed in testis, Proceedings of the National Academy of Sciences of the United States of America 91 (1994), 12952-12956.

[30] K. Kawakami, S. Noguchi, M. Noda, H. Takahashi, T. Ohta, M. Kawamura, H. Nojima, K. Nagano, T. Hirose, S. Inayama, H. Hayashida, T. Miyata and S. Numa, Primary structure of the alpha-subunit of Torpedo-californica $\left(\mathrm{Na}^{+}+\mathrm{K}^{+}\right)$-ATPase deduced from cDNA sequence, Nature 316 (1985), 733-736.

[31] W. Humphrey, A. Dalke and K. Schulten, VMD: Visual molecular dynamics, Journal of Molecular Graphics 14 (1996), $33-38$.

[32] J.C. Phillips, R. Braun, W. Wang, J. Gumbart, E. Tajkhorshid, E. Villa, C. Chipot, R.D. Skeel, L. Kale and K. Schulten, Scalable molecular dynamics with NAMD, Journal of Computational Chemistry 26 (2005), 1781-1802.

[33] A.K. Mandal, L. Mikhailova and J.M. Arguello, The Na,K-ATPase S5-H5 helix - structural link between phosphorylation and cation-binding sites, Annals of the New York Academy of Sciences 986 (2003), 224-225.

[34] J.B. Koenderink, S. Geibel, E. Grabsch, J.J.H.H. De Pont, E. Bamberg and T. Friedrich, Electrophysiological analysis of the mutated Na,K-ATPase cation binding pocket, Journal of Biological Chemistry 278 (2003), 51213-51222.

[35] R. Chenna, H. Sugawara, T. Koike, R. Lopez, T.J. Gibson, D.G. Higgins and J.D. Thompson, Multiple sequence alignment with the Clustal series of programs, Nucleic Acids Research 31 (2003), 3497-3500.

[36] J. Underhaug, L.O. Jakobsen, M. Esmann, A. Malmendal and N.C. Nielsen, NMR studies of the fifth transmembrane segment of $\mathrm{Na}^{+}, \mathrm{K}^{+}$-ATPase reveals a non-helical ion-binding region, FEBS Letters $\mathbf{5 8 0}$ (2006), 4777-4783.

[37] G. Nielsen, A. Malmendal, A. Meissner, J.V. Moller and N.C. Nielsen, NMR studies of the fifth transmembrane segment of sarcoplasmic reticulum $\mathrm{Ca}^{2+}$-ATPase reveals a hinge close to the $\mathrm{Ca}^{2+}$-ligating residues, FEBS Letters 544 (2003), $50-56$.

[38] H. Georg, A. Barth, W. Kreutz, F. Siebert and W. Mantele, Structural-changes of sarcoplasmic-reticulum Ca ${ }^{2+}-$ ATPase $^{-}$ upon $\mathrm{Ca}^{2+}$ binding studied by simultaneous measurement of infrared absorbance changes and changes of intrinsic protein fluorescence, Biochimica et Biophysica Acta - Bioenergetics 1188 (1994), 139-150.

[39] A. Barth, Phosphoenzyme conversion of the sarcoplasmic reticulum $\mathrm{Ca}^{2+}$-ATPase - Molecular interpretation of infrared difference spectra, Journal of Biological Chemistry 274 (1999), 22170-22175. 


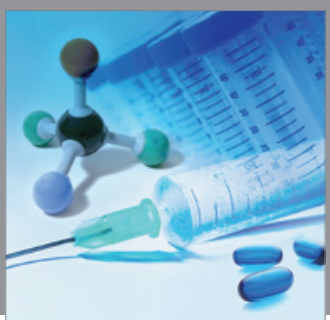

International Journal of

Medicinal Chemistry

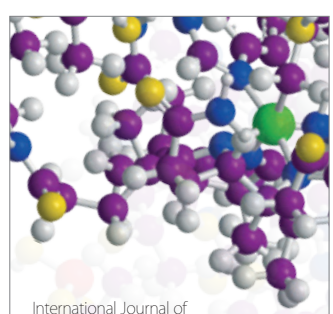

Carbohydrate Chemistry

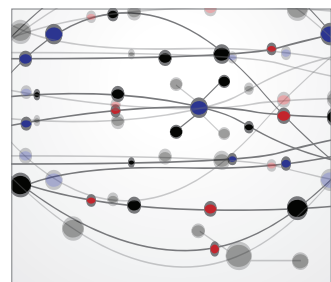

The Scientific World Journal
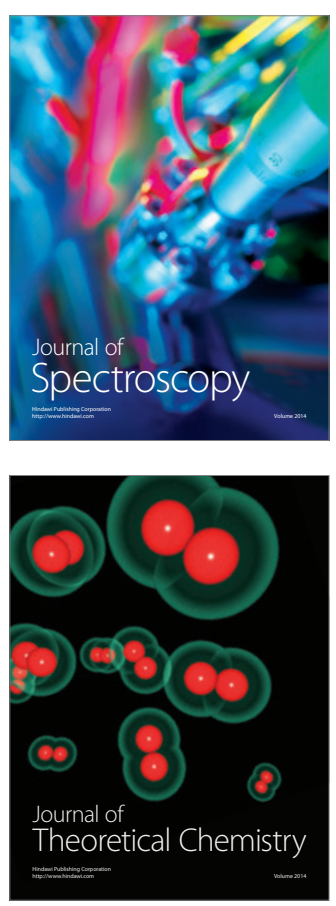
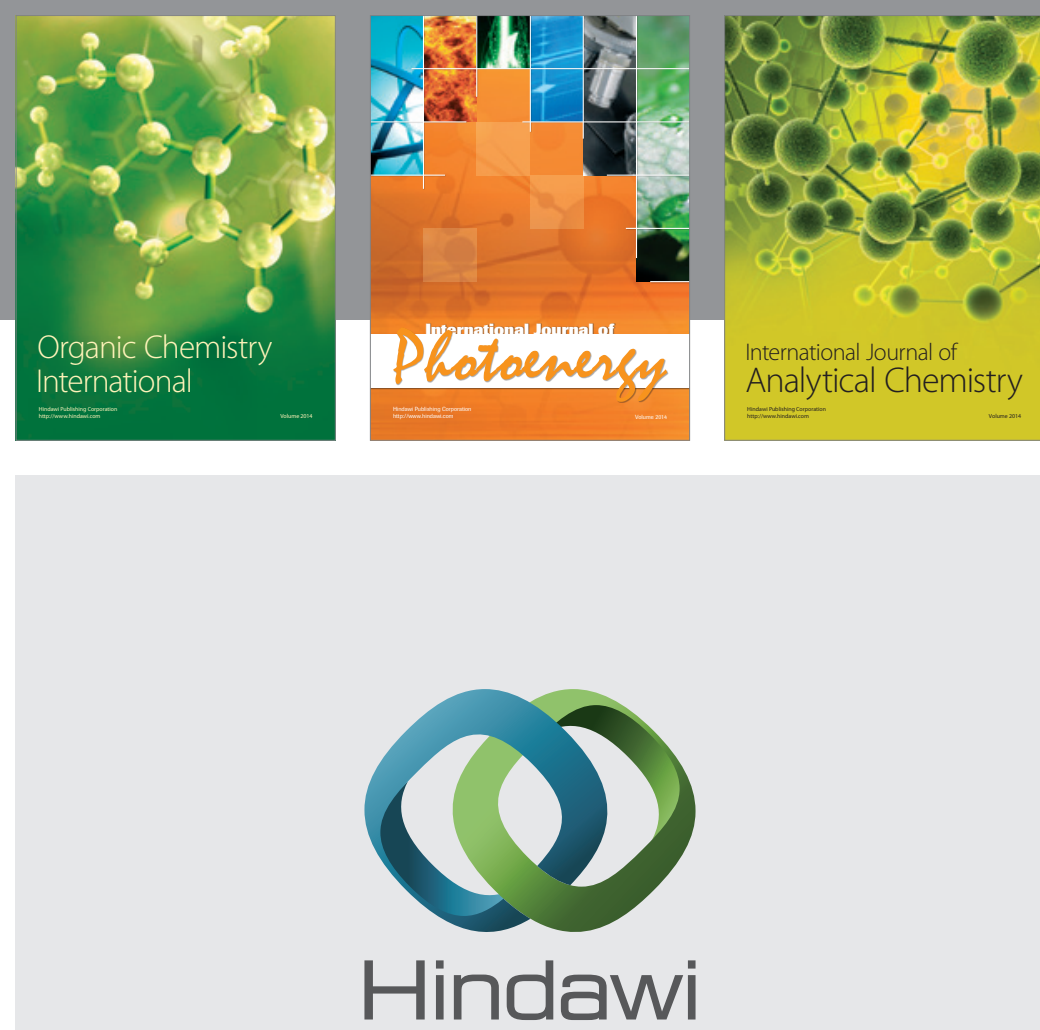

Submit your manuscripts at

http://www.hindawi.com
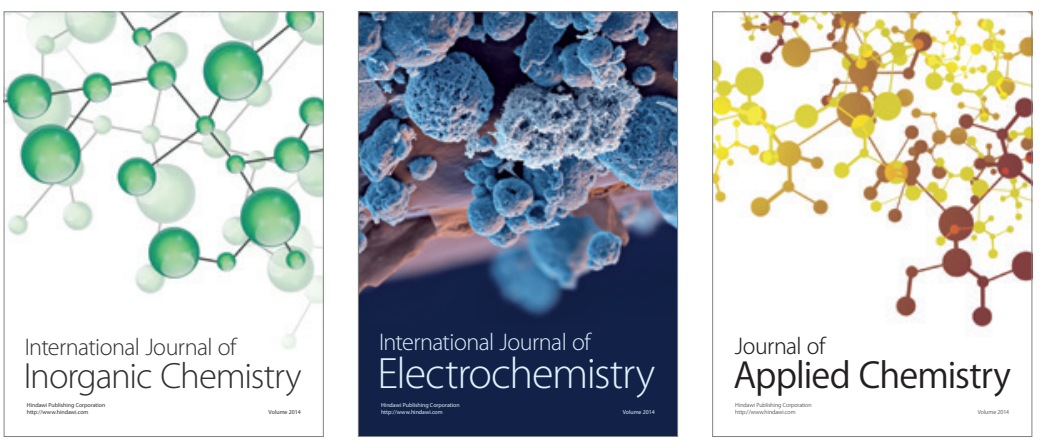

Journal of

Applied Chemistry
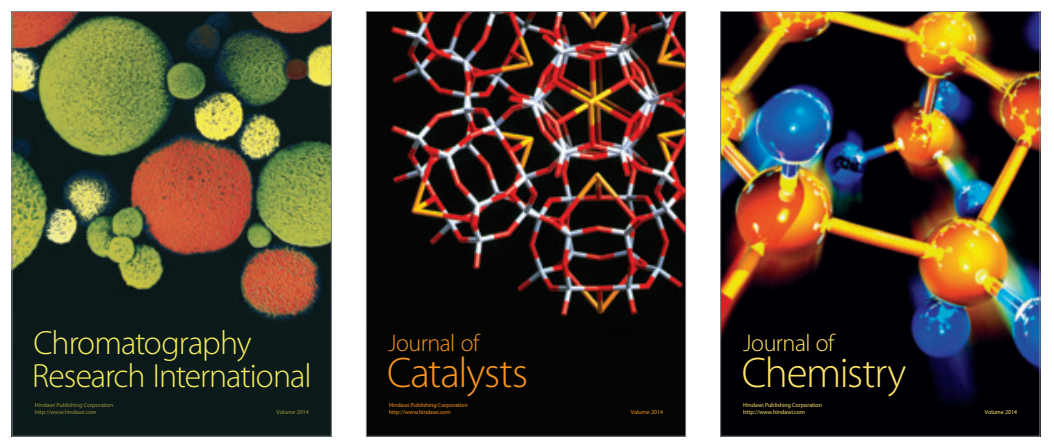
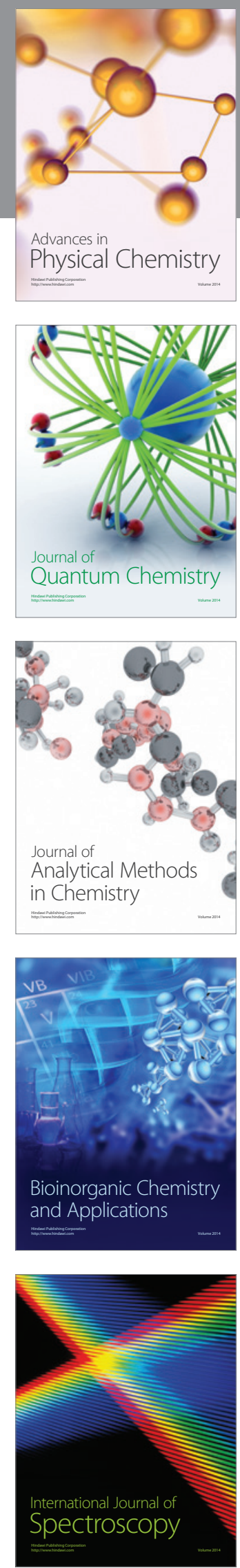